\title{
Compartmentalizing Web Browsing with Sailboat
}

\author{
Minchu Kulkarni \\ Ashoka University \\ Sanchit Bansal \\ Ashoka University \\ sanchit.bansal@alumni.ashoka.edu.in
}

minchu.kulkarni@alumni.ashoka.edu.in kshitij.kapoor@alumni.ashoka.edu.in

\author{
Deva Surya Vivek Madala \\ Ashoka University \\ deva.madala@alumni.ashoka.edu.in
}

\author{
Sudheendra Hangal \\ Ashoka University \\ hangal@ashoka.edu.in
}

\begin{abstract}
Users of web browsers today swim in a sea of haphazardly organized tabs, bookmarks, searches and downloads. This is because browsers operate at the level of individual web pages, and rarely understand the user's high-level tasks. We propose to address this problem by compartmentalizing the web browsing experience across different tasks defined by the user.

We describe Sailboat, an extension for Google Chrome that explores this idea by making user-defined tasks first-class objects in the browsing experience. In Sailboat, different tasks have their own tabs, history, bookmarks and download folders, while maintaining a unified identity across compartments. Sailboat lets the user create and switch between tasks such as "Philosophy Paper", "Summer Internships", and "Trip to China". Users can encapsulate the browsing related to a task, archive it or share it with others, and come back to it months later. Sailboat also tracks and reflects the analytics of time spent on different tasks to aid productivity.

We deployed Sailboat with several users, and studied how they engaged with it. Our users report that Sailboat aids organized browsing, reduces distractions, and is easy to get used to.
\end{abstract}

\section{CCS CONCEPTS}

- Information systems $\rightarrow$ Browsers; • Human-centered computing $\rightarrow$ Web-based interaction.

\section{KEYWORDS}

Web browsers, tab clutter, browsing history, personal information management.

\section{ACM Reference Format:}

Minchu Kulkarni, Kshitij Kapoor, Deva Surya Vivek Madala, Sanchit Bansal, and Sudheendra Hangal. 2019. Compartmentalizing Web Browsing with Sailboat. In India HCI 2019 (IndiaHCI '19), November 1-3, 2019, Hyderabad, India. ACM, New York, NY, USA, 8 pages. https://doi.org/10.1145/3364183. 3364187

Permission to make digital or hard copies of all or part of this work for personal or classroom use is granted without fee provided that copies are not made or distributed for profit or commercial advantage and that copies bear this notice and the full citation on the first page. Copyrights for components of this work owned by others than ACM must be honored. Abstracting with credit is permitted. To copy otherwise, or republish, to post on servers or to redistribute to lists, requires prior specific permission and/or a fee. Request permissions from permissions@acm.org.

IndiaHCI '19, November 1-3, 2019, Hyderabad, India

(C) 2019 Association for Computing Machinery.

ACM ISBN 978-1-4503-7716-4/19/11 . .\$15.00

https://doi.org/10.1145/3364183.3364187

\section{INTRODUCTION}

The internet has evolved tremendously since its inception and so have web browsers. It would not be an overstatement to say that browsing the internet has become an integral part of our lives. A large portion of web browsing is aimed at completing disparate tasks, such as searching the web before planning a trip, checking reviews online before watching a movie, watching videos on YouTube before buying a new phone, using online spreadsheets and documents for coursework, and so on. Most of these activities are done using a web browser, but current browsers do not provide much support for task oriented browsing.

Although browsers have evolved to become more secure, fast and efficient, they have not adapted well to the consumption of the large amount of information that a user now consumes. A significant improvement in the user interface of web browsers was the introduction of browser tabs. Tabs served the needs of managing multiple pages while browsing. Apart from facilitating rapid switching between pages, they are used in a wide variety of ways, from acting as reminders, to loading pages in the background, to working as short term bookmarks [4].

\subsection{Problems of today's browsers}

Along with their advantages, tabs have given birth to new problems of their own. Multitasking between various work and personal projects using a web browser has become common. Users often work simultaneously on these tasks, switching back and forth between tabs related to them. The ease of using tabs means that tens or even hundreds of tabs may be open at a time, leading to tab clutter. Tabs capture the "working set" of pages across many different tasks; they have a lifetime of a few hours or a few days, during which they must be kept open because they retain context to which users may want to return. We view the problem of tab clutter as a problem of short-term information management. In an initial survey we conducted across 75 participants, $50.7 \%$ responded that they considered tab clutter a problem. Further, a large proportion of people $-50.3 \%$ for history and $61.3 \%$ for bookmarks - rarely found history and bookmarks to be useful for re-finding information ${ }^{1}$.

Tab clutter not only makes finding pages in the working set difficult and inefficient, but also creates the problem of distraction. Tabs unrelated to the current task catch a user's attention since they are always visible to the user and may cause users to drift between tasks. This is similar to how users drift on websites like news, video

\footnotetext{
${ }^{1}$ All data and code for this paper, including the detailed responses of this survey, are provided as supplemental material with this paper.
} 
streaming and social networking sites. (The strength of recommendation engines ensures that users get distracted and indulge in often unintended binge-watching or reading.) Similarly, users find their browser history, downloads folder, and bookmarks cluttered with information across different tasks. This leads to difficulty in re-finding useful information, lack of fire-walling between tasks, and difficulty of preserving work done in a particular task.

\subsection{Task-based browsing and Sailboat}

We envision a future where browsers are redesigned to facilitate higher-level user tasks and improve the user's productivity by helping them avoid distractions and stay focused on the task at hand. If the browser were aware of the tasks the user is working on, pages could be presented in smaller, contextually relevant groups that are easy to process and interact with.

To explore these ideas we built Sailboat, a Google Chrome extension that enables task-based browsing. In Sailboat, users manually create tasks for various things they are working on such as "CS106", "Trip to China", and "Buy phone". Sailboat compartmentalizes tabs, bookmarks, browsing history, analytics, archived pages and the downloads folder across these tasks. All tabs belonging to a task are kept in a single window at all times. New tabs open in the same window, and thus belong to the same task. Users can move pages easily between tasks if necessary. Tasks may be in the foreground or background.

Our design of Sailboat takes inspiration from the desktop interface that users are already familiar with. Sailboat inserts a collapsible dock on every page using which users can interact with tasks (See Fig. 1). This dock shows available tasks and offers interactions to close or create tasks, providing users a familiar mental model and making it easy to get used to task-based browsing. Open tasks are visually differentiated, similar to the Mac OS Dock or the Windows taskbar. In addition, Sailboat provides analytics about the time spent on various tasks.

Sailboat makes it easy to re-find useful information. Since the user only sees the tabs from the current task, the user doesn't have to sift through several irrelevant tabs to locate the right one. However, if she wants to multitask, she can still do so by switching between browser windows. Web page history is recorded separately for each task along with time spent on each page. Files downloaded when in a particular task are saved in a folder dedicated to the task, keeping the downloads folder organized and making it easier to quickly find a downloaded file when required. Since only those tabs and bookmarks that are relevant to the current task are shown in the current browser window, users also tend to get less distracted by information in other tasks.

Sailboat also introduces a simple way to archive pages under the current task, and lets users create their own 'personal web' of the information they have found useful. Users can search through the full text of the page content of all the archived pages to easily re-find information.

Once a task is complete, it can be archived. This means that the task will not be shown in the dock, but all of its tabs, history, bookmarks, archived pages are saved, thus preserving all the work and research done for that task. Furthermore, a task, active or archived, can be downloaded and shared with someone else, giving the users the benefits of the work done instantly. A professor could use this feature to archive all the research work done while planning a course, and revive it the next year, or a student could save the work done while planning a trip and share it with their friends who are visiting the same place many months later.

Our major contributions in this paper are the following:

- We propose a system of task-based browsing for web browsers which takes inspiration from the model of desktop that users are already accustomed to.

- We describe specific user-interface elements we have designed in response to observing users that make Sailboat effective.

- We report the findings from a user study of 20 users using Sailboat for seven days, and describe how users reacted to its various features.

The rest of this paper is organized as follows. The next section lists related work and contrasts it with Sailboat. We then provide a detailed description of Sailboat's features, along with a rationale for design choices. Thereafter, we describe our learnings from multiple rounds of user studies, discuss some limitations and our plans for improving Sailboat, and then present our final conclusions.

\section{RELATED WORK}

The need for task based browsing has been felt for almost a decade: In 2010, Dubroy and Balakrishnan reported that people often used multiple windows in conjunction with tabs to create higher level task-based groups [4]. The study further reported that users often opened tabs that are related to multiple tasks together. Also in 2010, Rajamanickam et al. created a prototype of a task-based browser called Tab Four that allowed users to work on different tasks, all having their separate set of tabs and bookmarks [12]. Sailboat is based on similar ideas but uses more comprehensive compartmentalisation and a completely different design for the user interface. In addition to tabs and bookmarks it also compartmentalises entities such as history, time spent, and archived pages. The authors also compared Tab Four with Firefox in a study with 8 participants. They did not see statistically significant differences in task load comparisons; however, the participants did find task-based browsing to be useful, and reported that they would use it if it was in their primary browser. This study was helpful in reaffirming our belief in task-based browsing.

Ahuja's 'Contextinator' also explored ideas related to task based browsing [1]. However, its primary focus was on addressing the problem of information fragmentation due to different online services such as Gmail, Dropbox and Google Drive.

In 2018, Sohn and Gehrig explored the problems of tab management, re-finding information and context sensitivity by creating a concept design for a Safari based browser that allowed users to create 'spaces' [7]. Task based approach to history was explored in 2001 by Matthias Mayer [8]. Rybakov's insightful article on open tabs as cognitive spaces reinforces the need for tab collections that could be saved, archived, backed-up and restored [14].

Commercial tools such as Ghost Browser [3], and Workona (which has 49,611 users as per Chrome Web Store as of September 2019) [18] have also explored task-based browsing. Ghost Browser has an interface for creating tasks and sub-tasks, switching between tasks and archiving tasks. Workona allows users to create 

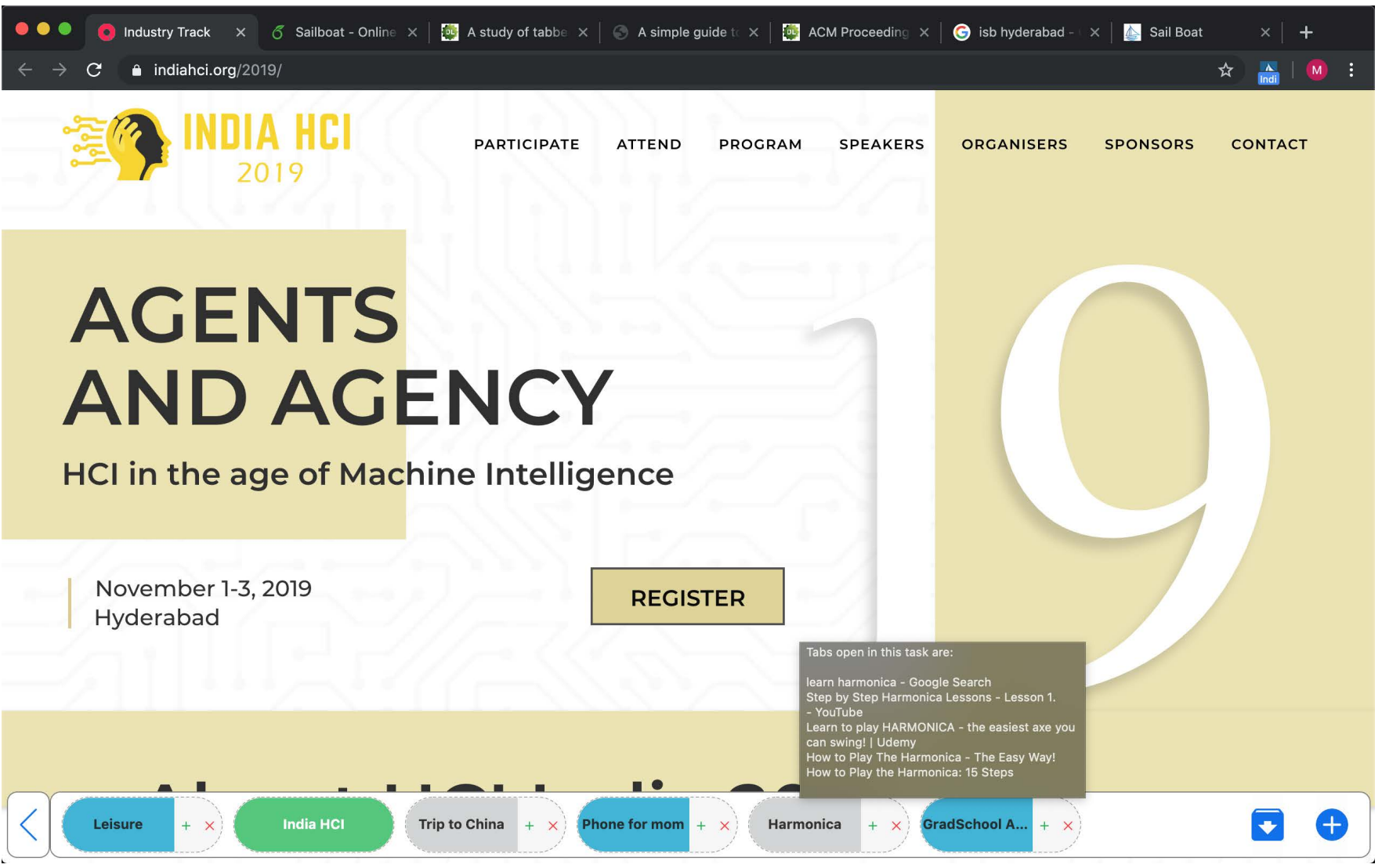

Figure 1: Sailboat dock

A screenshot of Sailboat on the home page of the India HCI 2019 conference. The 'India HCI' task is the task open in the current window. Tasks with blue background are open, but in backgrounded windows. The 'Harmonica' task is hovered upon and the tabs in that task are visible in the tooltip. Clicking the '+' button adds the current page to the respective task. Clicking on the ' $\mathrm{X}$ ' icon of an open task closes it. The 'Archive' and 'Create new task' buttons are seen in the dock on the right. The dock can be collapsed or expanded with the blue arrow button on the left.

'workspaces' that are similar to tasks. While both can be used for task-based browsing, unlike Sailboat, they only help in managing active tabs and do not compartmentalize history, bookmarks, and downloads, nor do they track time spent on various tasks. Moreover, their users have to shift to a completely different browser which might not be acceptable to many people. Sailboat's deployment model of a Chrome browser extension has a better chance of widespread adoption.

Browsers such as Google Chrome support user profiles, which are useful to manage separate identities [6]. A Chrome profile is like a different instance of the browser with the same functionality but has its own history, bookmarks, cookies and even settings. This extreme compartmentalization, however, brings its own set of problems such as the inability to share extensions and preferences (such as themes, saved passwords, and visited links) across profiles. Therefore, a user would have to separately install all the extensions, save all the passwords, and set other preferences across profiles, amounting to a cumbersome effort that would rarely be carried out by users. It is also not possible to compare analytics about browsing activities across profiles easily.
There are several popular tab management extensions like OneTab [10], Toby [17], and Sessions Buddy [15] which allow users to create tab groups. Onetab currently has over 2 million users and its popularity indicates that users are seeking a solution to the problem of tab clutter. Tab groups managed by these extensions are different from Sailboat tasks. Tab groups are passive objects, like folders. If a user wants to add or remove a tab from a group, she needs to do it explicitly on the extension's page. Sailboat tasks, on the other hand, are active objects. A Sailboat task is modified automatically when a user opens or closes a tab while browsing. These extensions focus on tabs and do not compartmentalize bookmarks, history or downloads and thus do not fully support task-based browsing.

Ophir et al explored cognitive control in media multitaskers and reported that heavy multitaskers were more susceptible to getting distracted [11]. Sailboat therefore incorporates features such as analytics about time spent in different tasks to help users understand their own browsing and time allocation better. The popularity of tools like Rescuetime [13], WebTime Tracker [16], StayFocusd (Over 750,000 users per the Chrome Web Store) [9], and the introduction of features like Screen Time and App Limit in iOS 12 [2] and Google Well 
Being tools in Android Pie [5] are also evidence that people care about analytics about their digital activity on and off the browser. With Sailboat, users can see the amount of time spent on a page or on a task. While some Chrome extensions do show time spent on a page or a particular domain, the user has no way of knowing how much time was spent on a particular task.

\section{TASK-BASED BROWSING WITH SAILBOAT}

When designing Sailboat, we initially conducted a formative survey of university students and professors to understand their problems with current browsers. We received 75 responses. Table 1 below summarises the important insights.

\begin{tabular}{|l|l|}
\hline Characteristic & \% Respondents \\
\hline Complained about tab clutter & $50.7 \%$ \\
\hline Did not use folders for bookmarks & $38.7 \%$ \\
\hline $\begin{array}{l}\text { Used a few unorganised bookmarks fold- } \\
\text { ers }\end{array}$ & $44.0 \%$ \\
\hline Did not review browser history & $53.3 \%$ \\
\hline
\end{tabular}

Table 1: Formative survey results

In addition, $54.7 \%$ of respondents said that they would find it useful to separate their browsing history, bookmarks and downloads according to tasks or themes.

From this initial survey, we realized that compartmentalizing web browsing would enhance the browsing experience. We reviewed all browser functions in the context of compartmentalization across tasks. To ensure that the learning curve for working with these compartments is minimal, we designed the interaction to be similar to that of Windows or Mac OS X desktops. For example, users can create, switch, and close tasks through a dock that emulates the already familiar OS dock.

\subsection{Tasks in Sailboat}

With Sailboat, the user is always browsing in the currently active task. Tasks are first class objects that have their own tabs, bookmarks, history, downloads folder, archived pages, and once active, their own window. By default, the user is in a default task called 'Leisure'. This is meant for general browsing when the user is not working on any particular task.

A user can create, switch, close, archive, and delete a task. A newly created task is a window with just one tab (the new tab), and no bookmarks. The user can now browse in this task, opening as many tabs as needed. On each tab/bookmark creation, removal or update, the task is updated. The history of pages for this task is also updated as the user browses the web. A separate folder is created in the browser's 'Downloads' folder for each task. When a file is downloaded, the corresponding task folder is used by default, unless the user specifically overrides it. This feature makes it easy to save the file in the task-specific folder, keeping the Downloads folder more organized. This feature also helps when the user is looking for a file as she knows which folder to look in.

When the user switches to a different, unopened, task, a new window is opened with the tabs and bookmarks of that task. Upon closing the task, the window closes. If the user quits the browser, all the windows (and therefore, the open tasks) will close. However, the task state is retained by Sailboat and will be restored when the browser re-opens and a task is reactivated. Similarly, addition and deletion of bookmarks are also recorded in the task. Hence the tabs and bookmarks of the task are sustained over all these actions. When the user reopens or switches to a task, they sees a window that is set up exactly like they had left it.

A task can be in one of four states - currently active; open, but backgrounded; unopened; and archived. The current task, as the name suggests, is the task that is open and is in focus. There is only one current task at any given point. The current task is shown with green background in the dock. A task is open in the background when it has been opened but its window is not in focus. Such tasks are shown with blue background in the dock. Unopened tasks, shown with a grey background, are not open in any browser window. These tasks do not have a browser window open for themselves until the user chooses to 'open' them. Archived tasks are the ones that a user has closed or is not likely to revisit soon. These tasks are not shown in the dock. However, they are available below all the non-archived tasks in the new tab page.

Users occasionally drift between tasks and open a tab that is in the wrong task. If this happens, they can move a tab from one task to another by clicking on the ' + ' button shown beside each task on the dock.

When the user is done with a task it can be either archived or deleted. Tabs, history and bookmarks of an archived task are stored separately and the task will not be shown in the dock or on the new tab page. The task's data can, however, be retrieved later when needed, thus preserving the research/work done for the task.

\subsection{Compartmentalizing the browsing experience}

In Sailboat, both short-term information (like tabs), and long-term information (like history, bookmarks, downloads, and archived pages) are compartmentalized and assigned to user-defined tasks. As a result, users see information in small and coherent chunks, instead of having to deal with a large amount of unorganized information. This eases re-finding and processing of information. Clearly, locating a tab from within 5 tabs is easier than finding a tab from 30 tabs. Similarly, finding a bookmark or a downloaded file is quicker merely because there is lesser information to sift through. Reviewing a coherent task-based history is more efficient and effective than going through cluttered browser history.

\subsection{User interface that emulates the desktop}

From our preliminary testing, we learned that the interface for managing tasks was as important as the idea of tasks. In an early version of Sailboat, the user had to click on the extension icon beside the Google Chrome Omnibox to interact with a task (i.e., to create, open, close, archive, add or delete tabs to a task). Although users could see the current task on the extension icon badge, the task visibility was limited. Users also had to make an extra click every time they wanted to switch or open a task. Our initial testers told us that they either forgot to create tasks altogether, or they forgot to switch to a task at the appropriate time because all their tasks were not always visible. In short, they forgot that they had Sailboat. 
To address this problem, we moved this interaction to a collapsible 'dock' which Sailboat injects and displays on each page (See Fig. 1). The dock solves the problem of task visibility along with removing the extra click that was involved in the popup based interface. The Sailboat dock is designed to emulate the MacOS dock or Windows Taskbar (see Table 2 for parallels). The dock also allows users to move tabs from one task to another, and add links to another task by dragging a link and dropping it on the desired task. The dock can also be used to create new tasks and archive pages. It can be minimized if the user does not need it, or if it interferes with the content at the very bottom of the page.

Sailboat users have found very useful the ability to add tabs to another window without having to switch to the window. Similarly the ability to close a window without actually being in it has also been found to be useful.

\begin{tabular}{|l|l|}
\hline OS Dock/Taskbar & Sailboat Dock \\
\hline Open/Close applications & Open/Close Tasks \\
\hline $\begin{array}{l}\text { See windows of an application } \\
\text { on hover }\end{array}$ & See tabs of a Task on hover \\
\hline Open file by dragging onto App & $\begin{array}{l}\text { Add link by dragging onto a } \\
\text { Task }\end{array}$ \\
\hline See if an App is open & See if a Task is open \\
\hline $\begin{array}{l}\text { Cmd/Ctrl+Tab Shortcut to } \\
\text { switch between applications }\end{array}$ & $\begin{array}{l}\text { Ctrl+ Shortcut to switch be- } \\
\text { tween Tasks }\end{array}$ \\
\hline
\end{tabular}

Table 2: Parallels between OS Dock and Sailboat Dock.

Users can rapidly switch between tasks through a shortcut (Ctrl+ ); this is similar to the OS shortcut of switching between applications.

Another way to interact with tasks is through the new tab page. Sailboat replaces the new tab page with a page where users can open, close, rename, archive, or delete tasks. The time spent on each task is also shown on the task cards. Thus, this page provides a birds-eye view of all tasks. With the navigation bar at the top of this page, users can navigate to task-based history, Sailboat search, task dashboard, settings, about and help pages.

\subsection{Task Awareness and Reducing Distraction}

An advantage of increased task visibility is the increase in task awareness. Since users can see the tasks that they need to work on (in the dock or on the new tab page), almost all users reported having become more aware about the task that they had set out to do. They tended to refrain from opening tabs related to other tasks. Moreover, since they do not open tabs related to other tasks, they only see tabs and bookmarks of the particular task, thus avoiding distraction.

Sailboat also facilitates overall browsing awareness by showing a graph of the time spent per task in its dashboard. The graph has dates on the $\mathrm{x}$-axis and time spent on the $\mathrm{y}$-axis. For each date there is a column for all the non-archived tasks on that day. Users can easily filter the time period to look at the time spent per task for current day, week, fortnight or month.

\subsection{Creating and Searching a Personal Archive}

Through our initial surveys, we realized that users needed a way to mark pages as useful and perhaps have the capability of searching through the full page content of such pages. This capability is not supported by browsers today; ironically, users find it easier to search the whole web than their own history. We therefore introduced a way to archive pages. The user can archive pages they found even mildly interesting or useful. The user may not even have completely read by the page. But by clicking on the archive button in the dock (or using a shortcut), they can import it into their own personal slice of the web. It is important to note that archiving a page is different from bookmarking it. Bookmarks can be thought of as long-term pointers to useful information, while archived pages store the content of potentially useful pages. This information, unlike bookmarks, can then be easily searched, surfaced locally or used for unforeseen purposes in the future. In a sense, this is like 'liking' a page - it constitutes a mild endorsement, but for one's own future use rather than to refer it to friends. Users can build a curated personal web of pages they have endorsed that will potentially be of use in the future. For example, archive search results could be plugged into the user's web search results page, so that the user can search within their personal web and the global web simultaneously.

\section{USER STUDY}

We conducted several rounds of surveys, pilots, and user studies to inform the design of Sailboat. As described earlier, before starting the design of Sailboat, we sent out a formative survey to students and professors on our campus, asking if they faced problems related to browsing, such as tab clutter, re-finding of information, and distractions. After developing the initial version, we conducted informal preliminary testing. These tests informed our second version of Sailboat, which we gave out for pilot testing. After incorporating feedback from the pilot study, we conducted the final user study. In this section, we report the results from the pilot and the final user study.

\subsection{Methodology}

4.1.1 Pilot. After running the initial informal tests, we implemented several refinements and were ready to test an updated version of Sailboat. Before the final user study, we ran a pilot test with 8 users from our university campus for 5 days (participants aged 19-26 years, mean $=21.2$ years, S.D. $=2.3$ years, 6 males and 2 females, majoring in Computer Science, Psychology, International Relations, English, and Economics) Out of our 8 pilot users, no one had used an extension similar to Sailboat in the past. Each user reported spending at least five to six hours per day on their browser. The users also filled diary entries (in the form of a Google form) at the end of each day on their own computer. The diary entry consisted of questions about the kind of browsing tasks they worked on, and how they used the different features offered by Sailboat. The pilot users were not given any incentives. The pilot study provided a glimpse of the likely usefulness of the various features of Sailboat.

During the pilot, we found and fixed various types of bugs on different platforms. We also received useful feedback that helped us make some changes before moving into the actual user study. For example, our default task was initially called 'Default'. One of the participants created a task called 'Time-Wasting' to open all her entertainment and non-work related pages (primarily in order to discourage herself from using it!) This gave us the idea to change the 


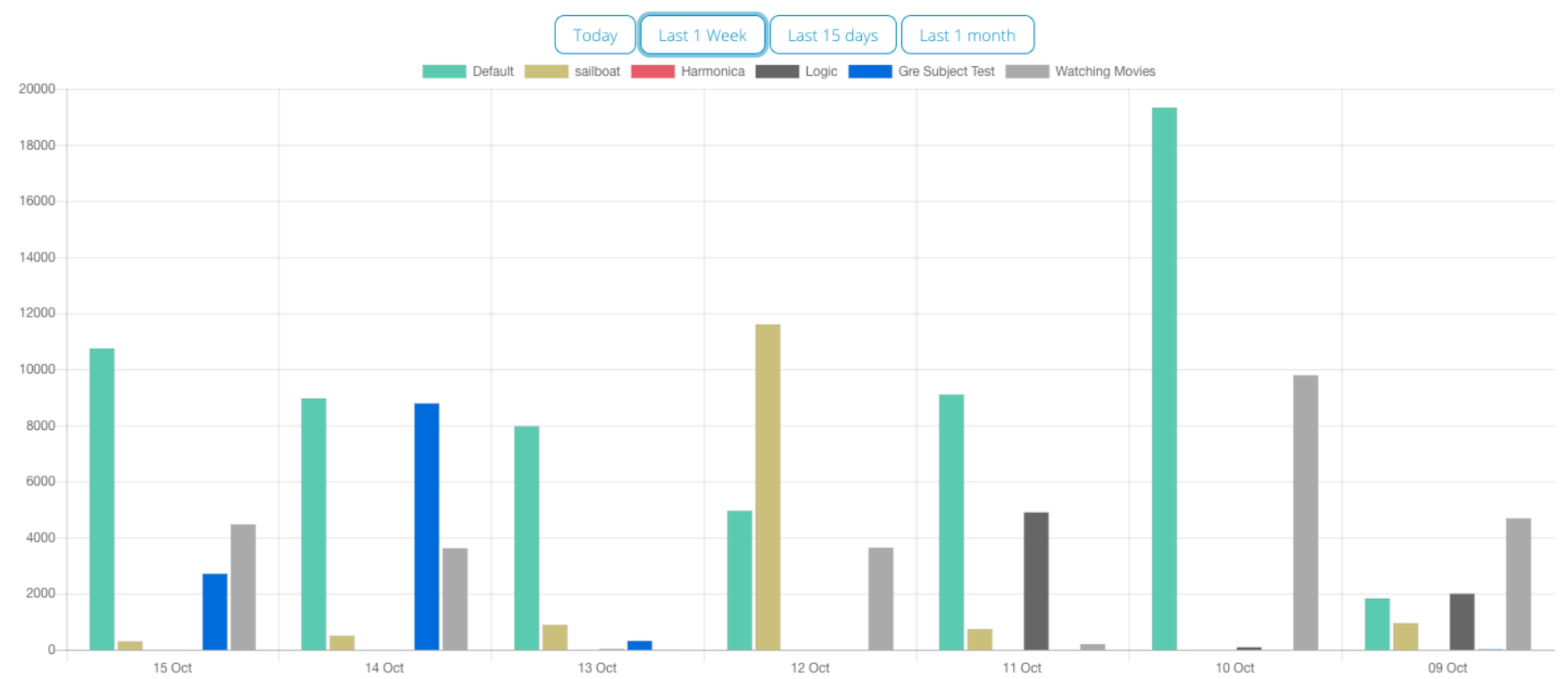

Figure 2: Dashboard of time spent per task

A screenshot of Sailboat's dashboard that shows time spent on each task over the selected time period. Users can choose one of the following - Today, Last 1 Week, Last 15 Days, Last 1 Month. Dates are on x-axis and time spent (in seconds) is on y-axis. Hovering on the columns shows time spent in minutes.

name of the Default task to "Leisure", as it makes people explicitly aware of being in a work or leisure task, and encourages them to create more work-related tasks. Out of the eight pilot users, seven continued using Sailboat and provided us useful feedback after the pilot as well.

4.1.2 Final User Study. Next, we report on a larger user study we conducted to test a more refined version of Sailboat. We recruited participants via email by inviting people to respond to posters placed around our campus. The respondents had to fill out a form asking about their web usage (the web browser used, average daily usage, etc.) prior to becoming a part of the study. The form allowed us to filter out respondents who were not Google Chrome users. Eventually we signed up 20 participants for the study (ages 17-24 years, mean $=19.8$ years, S.D. $=1.5$ years, 11 males $/ 9$ females, majoring in Computer Science, Psychology, Political Science, Physics, English, Economics, Finance, and Biology). We will refer to them as U1 to U20. Of these 20 people, U4, U7 and U11 had used a different extension for tab management prior to the study. Every participant had already installed at least 3 Google Chrome extensions prior to installing Sailboat. We gave all participants an initial demonstration for 30 minutes and a walk-through of each feature of Sailboat. They used Sailboat for 7 days, and filled out a diary entry (in the form of a Google form) each night on their own computers. ${ }^{2}$. Four days into the study, we also conducted two focus groups. The aim of the

\footnotetext{
${ }^{2}$ The data for these diary entries is available in Final_User_Study.csv in supplementary materials.
}

focus group was to gain qualitative insight into the usage of Sailboat. There were two focus groups with 10 users each. Participants were compensated with an Amazon gift coupon worth ₹ 500 (about US\$7).

\subsection{Qualitative feedback}

Through the focus group and diary entries, our primary finding was that Sailboat was effective in reducing distraction levels. Using Sailboat, users transferred the tabs that were unrelated to the current task into other tasks, and continued working without moving from the current window or losing those tabs.

In the focus group interview, U15 remarked, "Sailboat helped me focus attention on reading task related articles. I added the other tabs to a new task and read them later."

Through the diary entries, we saw a gradual decline in the reported distraction level of the users. This was measured on a Likert scale going from 1 for 'not distracted' to 5 for 'very distracted'. The Likert averages monotonically decreased from 2.7 to 2.05 from the first to the fourth day. On the fifth, sixth, and seventh days the Likert averages were $2.2,2.1$, and 2.3 respectively. We suspect that the slight increase was due to the weekend starting on the fifth day. Through the week, we also observed a fall in the number of times users worked in the wrong task - it decreased almost monotonically from $43.8 \%$ on the first day to $15.8 \%$ on the fourth day. U1 said " Using Sailboat has improved my working method. It is less cluttered, more organized and helps me focus on the current task. Using it regularly will help me reduce the number of times I work in the 
wrong task." A user remarked that Sailboat helped them become aware about their tasks and reduced distractions. For example, a diary entry by U4 observed, "Since I wasn't disturbed with any tabs that contained anything but details about the task itself, I didn't get distracted quickly."

Sailboat's analytics features were found to be useful for maintaining accountability, and analyzing one's own usage. The time spent feature was found useful by more than half of all users on all seven days. U7 said "It keeps me accountable for the time I spend on each task. Earlier, I thought I did not do enough when it comes to career prep or university research or studying for class. This helps me see that I actually spend a lot of time doing things I should be doing." $\mathrm{U} 12$ remarked "I could segregate the time I spent on reading papers for academic purposes and doing other random tasks." U11 used the time spent feature in a unique way. Using Sailboat history, she sorted the pages by time spent and cited the pages on which she spent more than 20 minutes. Her diary entry reported: "Managed to get a lot of citation work done using the time spent per tab in history."

In the first focus group, we got an unexpected request from U3 and U9 asking us to add an option to temporarily disable Sailboat as it made them feel like they couldn't relax because they were always worried about their tasks. In the second focus group, U13 reported that he had a laptop with low specifications and hence he found the feature of adding tabs to an unopened task useful.

Some users in the focus group found the search results from browser history and archived pages displayed by Sailboat to be helpful, but other users felt that they need to use Sailboat for a longer time period to develop a rich enough history. U9 said "On searching for things, Sailboat showed me matching pages from my history. This was helpful in some instances."

\subsection{Quantitative results}

Apart from the diary entries at the end of each day, we also tracked tab- and task-related events with the participant's consent. We captured the tab ID, task ID and window ID whenever tab creation, tab activation, or tab removal were triggered. These events gave us a sense of the overall browsing pattern of a user. We did not capture personal data such as visited URLs or interaction of the user with the page.

The average number of tabs opened on Day 2 were 284.5. As we approached the weekend, on Thursday the figure dropped to 130.75 . Finally, on the weekend we had only 60.125 as our average. We also saw a similar trend in the number of task switches. On Day 2 we had 13.75 average task switches, Day 5 we had 9.56 and then on Day 7 we had 3.5. When asked, users reported that they used more tasks and tabs in the first part of the week; in the last part of the week, they were mostly working on just 2 or 3 tasks with fewer tabs. This indicated the necessity to factor in the rhythms and cadences of users' browsing patterns in our user study.

\section{LIMITATIONS AND FUTURE WORK}

The current implementation of Sailboat is not without limitations. A few important ones are listed below.

(1) Sailboat is available only for the Google Chrome browser. It is also not available on mobile and tablet devices.
(2) Sailboat is unable to track time spent outside of the browser. This means Sailboat analytics may not give a complete picture of the time spent per task.

(3) Users currently have to create and manage tasks manually. Although this keeps users in control, it also requires them to do some work. We are considering options to automatically detect when the user has embarked upon a new task or is drifting from an existing task.

We plan to extend Sailboat in the following ways.

(1) We are experimenting with dynamically inserting results from archived pages of the current task and history into the results page of a Google web search.

(2) We intend to show task suggestions to reduce the burden of switching tasks. If a user is distracted while browsing and opens a page unrelated to the current task, Sailboat could issue a notification to the user based on textual similarity of the page with pages in the task.

(3) We would like to consider a task-based evaluation experiment to capture metrics such as user satisfaction, task success, cognitive load, and time spent to finish the task.

(4) We can improve searching through archived pages by implementing an 'omnisearch' for the browser where results come from open tabs, archived pages, browser history, downloads folder etc.

\section{CONCLUSION}

In this paper we have described an effective way to improve browsing efficiency and reduce distraction. We have provided solutions to the problems of tab clutter, unusable browser history and bookmarks, distraction and difficulty in re-finding useful information. Sailboat, our Google Chrome extension, helped organize users' browsing based on the tasks they were working on. Our user study with 20 participants showed a decline in distraction over time. A majority of the users liked task-based browsing with Sailboat, found it easy to get used to, and said they would like to continue using it. We recommend that browser designers support task-based browsing directly in order to make web browsing more efficient.

\section{SUPPLEMENTARY MATERIAL}

All data related to this paper (such as survey responses, diary comments and user metadata files) are provided as supplemental material with this paper. However, any personally identifying information has been removed. To use this data, please see the instructions in the provided file README.txt. The source code for Sailboat is also available as part of the supplementary material. Updated versions of Sailboat may be found at https://github.com/hoominchu/sailboat.

\section{REFERENCES}

[1] Ahuja, A. Contextinator: Recreating the context lost amid information fragmentation on the web., Masters Thesis, Virginia Tech.

[2] Apple. iOS 12, Retrieved November 5, 2018.

[3] Browser, G. Ghost Browser 2018, November 5, 2018.

[4] Dubroy, P., and Balakrishnan, R. A study of tabbed browsing among mozilla firefox users. In Proceedings of the SIGCHI Conference on Human Factors in Computing Systems, CHI '10, ACM (New York, NY, USA, 2010).

[5] Google. Google Wellbeing, Retrieved November 5, 2018.

[6] Google. Google Chrome Profiles, Retrieved November 5, 2018.

[7] Julius Sohn, J. G. Refresh: Bachelor's Thesis. Hochschule fãijr Technik und Wirtschaft, Retrieved November 5, 2018. 
[8] M. Mayer, B. B. B. Browsing Icons: A Task-Based Approach for a Visual Web History. UMD HCIL '01 (2001).

[9] Media, T. StayFocusd (Chrome extension), Retrieved November 5, 2018.

[10] OneTab. OneTab extension for Google Chrome and Firefox, Retrieved November 6, 2018.

[11] Ophir, E., Nass, C., and Wagner, A. D. Cognitive control in media multitaskers Proceedings of the National Academy of Sciences 106, 37 (2009), 15583-15587.

[12] Rajamanickam, M. R., MacKenzie, R., Lam, B., and Su, T. A task-focused approach to support sharing and interruption recovery in web browsers. In $\mathrm{CHI}$
'10 Extended Abstracts on Human Factors in Computing Systems, CHI EA '10, ACM (New York, NY, USA, 2010), 4345-4350.

[13] Rescuetime. Find your ideal workâĂŚlife balance, Retrieved November 5, 2018.

[14] Rybakov, M. Open tabs are cognitive spaces, Retrieved November 5, 2018.

[15] sessionbuddy.com. Session Buddy, Retrieved November 5, 2018.

[16] Sittek, P. Webtime Tracker (Chrome extension), Retrieved November 5, 2018

[17] Toby. Better Than Bookmarks - Toby, Retrieved November 6, 2018.

[18] Workona. Workona, Retrieved November 5, 2018. 